\title{
MULTIDRUG-RESISTANT URINARY TRACT ISOLATES OF ESCHERICHIA COLI: FREQUENCY AND PATIENT DEMOGRAPHICS IN A TERTIARY CARE HOSPITAL.
}

1. MBBS, FCPS (Medicine) Assistant Professor of Medicine MTH, UMDC, Faisalabad.

2. MBBS, FCPS (Nephrology) Assistant Professor Nephrology MTH, UMDC, Faisalabad.

3. MBBS, FCPS (Gastroenterology) Associate Professor Gastroenterology MTH, UMDC, Faisalabad.

4. MBBS, FCPS (Medicine) Associate Professor Medicine MTH, UMDC, Faisalabad.

5. MBBS, FCPS (Medicine) Assistant Professor Medicine Mayo Hospital, Lahore.

6. MBBS, FCPS (Gynaecology) Assistant Professor Obstetrics \& Gynecology MTH, UMDC, Faisalabad.

7. MBBS

Medical Officer

Allied Hospital, Faisalabad.

Correspondence Address: Dr. Muhammad Ahsan Allied Hospital, Faisalabad. ahsanjahangir194@gmail.com

Article received on: 25/06/2019

Accepted for publication: 15/09/2019

\begin{abstract}
Salman Azhar 1 , Muhmmad Wasif Baig², Shahid Rasool ${ }^{3}$, Rizwan Rasool Khan ${ }^{4}$, Talha Munir ${ }^{5}$, Umber Fatima ${ }^{6}$, Muhammad Ahsan ${ }^{7}$

ABSTRACT: Urinary tract infections (UTIS) are a major burden to the health care as it is estimated that around 150 million UTIs occur yearly worldwide. Enterobacteriaceae are the most common agent causing serious urinary tract infections; and multi drug resistent (MDR) cases are increasing day by day. Objectives: To determine the frequency and patient demographics of multidrug resistant urinary tract isolates of Escherichia Coli in a Tertiary Care Hospital. Study Design: Retrospective cross sectional study. Setting: Department of Medicine, Madinah Teaching Hospital / The University of Faisalabad, Faisalabad. Period: May 2016 to Sep 2018. Material \& Methods: 187 patients of age 15 to 90 years with positive E. coli on urine culture and sensitivity were included in this retrospective cross sectional study. All those patients with history of dysuria (pain during urination) or frequent urination (more than 7 times per day) were advised urine complete analysis and those with $>5 \mathrm{WBCs}$ or pus cells /HPF or having positive for leukocyte esterase and/or nitrite, were advised urine culture and sensitivity. Main outcome variable was the frequency of MDR cases among the culture positive E. coli UTI patients. Results: Among all the cases of E. coli UTI, frequency of MDRE UTI was $66.8 \%(n=125)$ and rest $33.2 \%(n=62)$ cases were not MDR UTI. $97.3 \%$ patients were resistant to lactam antibiotics, $95.7 \%$ were resistant to quinolones and $68.4 \%$ were resistant to aminoglycosides. Conclusion: With the rise in the microbe's resistance to multiple drugs, new therapeutic strategies are to be designed after analyzing our population's susceptibility pattern. It is not less than a challenge for physicians of a developing nation like Pakistan to treat MDR E.coli UTI cases while taking care of the cost of treatment and controlling the disease burden at the same time.
\end{abstract}

Key words: $\quad$ Cystitis, E. Coli, Gram Negative, MDR, Muti-drug Resistance, UTI, Urine Infection, Urine Culture.

Article Citation: Azhar S, Baig MW, Rasool S, Khan RR, Munir T, Fatima U, Ahsan M. Multidrug-resistant urinary tract isolates of escherichia coli: Frequency and patient demographics in a Tertiary Care Hospital. Professional Med J 2020; 27(2):335-340. DOI: 10.29309/TPMJ/2020.27.2.3854

\section{INTRODUCTION}

Urinary tract infections (UTIs) are a major burden to the health care as it is estimated that around 150 million UTIs occur yearly worldwide. ${ }^{1}$ Urinary tract infection (UTI) is the fourth most common bacterial infection encountered by the physicians, occurring in all age groups and affecting both genders. About $20 \%$ of these infections occur due to gram positive microbes and more than $80 \%$ by gram negative microbes. ${ }^{2,3}$

Enterobacteriaceae are the most common agents causing serious urinary tract infections. These infections are also prevalent in Pakistan but with the development of antimicrobial resistance, MDR cases are increasing day by day. Choice of antimicrobial for treatment depends on the susceptibility profiles of the Enterobacteriaceae. ${ }^{4}$ As recommended by the Infectious Diseases Society of America, factors like local data of antimicrobial resistance, availability of antimicrobial under consideration and patient's history of drug allergies and tolerability profile should be kept under consideration. ${ }^{5}$

Nitrofurantoin or trimethoprim-sulfamethoxazole can be used for uncomplicated cystitis patients and quinolones, ceftriaxone, aminoglycosides, and carbapenems can be given as the empirical antimicrobial treatment for pyelonephritis/ complex UTIs. With the rise in multidrugresistant Enterobacteriaceae (MDRE), choice of 
antimicrobial has become a challenge for the treating physicians. ${ }^{6}$

Previously, resistance was reported for extendedspectrum beta-lactamases, now ending up in MDRE. $^{7}$ It has been reported that antibiotic resistance varies directly with the antibiotic abuse ${ }^{8}$, and MDR microbes are on the rise because of the excessive use of antimicrobials prescribed without investigating the nature and cause of disease, thus abusing them. ${ }^{9,10}$ Appropriate and prompt antimicrobial treatment of MDRE cases should be done to decrease morbidity and mortality. ${ }^{11}$

Prevalence of UTI is $11.6 \%$ among symptomatic patients in Pakistan, with trend approximately twice as high in females than males; and $80 \%$ of these are caused by gram negative rods (E.Coli UTI in $41 \%$ cases). ${ }^{12}$ As, limited local studies are available on the MDR E. coli urinary tract isolates ${ }^{13}$, the objective of our study was to determine the frequency and demographics of MDR E. coli urinary tract isolates among patient presenting in a tertiary care hospital in Faisalabad, Pakistan. The purpose of this study is to update all the health care members about the ongoing resistance pattern among the common infections like UTI, prevailing in the society.

\section{MATERIAL AND METHODS}

This was a Retrospective cross sectional study comprising 187 patients. All patients of either gender of age $>15$ years till 90 years having a positive urine culture and sensitivity for E.coli were included. A positive urine culture was defined as bacterial growth $\geq 10^{4} \mathrm{CFUs} / \mathrm{ml}$.

MDR E.coli cases were defined as those cases with resistance to two out of three antibiotic classes namely: (1) Beta-lactamase inhibitors (2) Fluoroquinolones (3) Aminoglycosides. Age, gender, urine WBC's, and results of urine culture and sensitivity were noted on a designed proforma. Main outcome variable was the frequency of MDR E.coli cases among the culture positive E. coli UTI patients.

All the variables were analyzed using SPSS 16. Nominal data were expressed as frequency and percentage while numerical data were analyzed and mean with standard deviation was calculated. To control the effect modifiers, data was stratified for age and gender and post-stratification chi square was applied to see the effect on the outcome. P-value of $<0.05$ was taken significant.

\section{RESULTS}

In this retrospective study, 187 cases of UTI with a positive culture showing E. coli were identified. Almost two third of these patients were females; $38 \%$ patients were male and $62 \%$ patients were females, with female to male ratio of about $2: 1$. Mean age was 51.48 years with a standard deviation of +17.44 , ranging from 18 to 90 years.

Among all the cases of E. coli UTI, frequency of MDR was $66.8 \%(n=125)$ and rest $33.2 \%(n=62)$ cases were not MDR UTI. (Table-I). Among these MDR E.coli UTI cases, $63.2 \%(n=79)$ were female and rest $36.8 \%(n=46)$ were males patients.

\begin{tabular}{|l|c|c|}
\hline \multicolumn{1}{|c|}{ MDRE UTI } & Cases & Percent \\
\hline Yes & $125 / 187$ & $66.8 \%$ \\
\hline No & $62 / 187$ & $33.2 \%$ \\
\hline
\end{tabular}

Table-I. Showing the percentage of MDR E.coli cases among the E. coli UTI cases

Among the total 187 cases, $97.3 \%$ patients were resistant to beta-lactam antibiotics, $95.7 \%$ were resistant to quinolones and $68.4 \%$ were resistant to aminoglycosides.

Effect modifiers like age and gender, were controlled through stratification and post stratification chi square test was applied. (TablesII and Table-III)

Age of the patients was stratified into young, middle, old. Those of 18 to 35 years were grouped into young category, 36 to 60 into middle age and 61 to 90 into old age group.

Post stratification chi square test was statistically significant for all categories of age but not statistically significant for gender. 


\begin{tabular}{|l|l|c|c|c|}
\hline \multicolumn{2}{|c|}{} & \multicolumn{2}{|c|}{ MDR E.coli UTIs } & Total \\
\cline { 3 - 5 } & & Yes & No & Yes \\
\hline \multirow{3}{*}{ Age } & Young & 22 & 17 & 39 \\
\cline { 2 - 5 } & Middle & 68 & 29 & 97 \\
\cline { 2 - 5 } & Old & 35 & 16 & 51 \\
\hline \multirow{2}{*}{ Total } & & 125 & 62 & 187 \\
\hline
\end{tabular}

Table-II. Post- stratification chi square applied for age in relation with MDR E.coli UTI cases

Chi square test $=2.45$-value $=0.293$

\begin{tabular}{|l|l|c|c|c|}
\hline \multicolumn{2}{|c|}{} & \multicolumn{2}{|c|}{ MDR E.coli UTIs } & Total \\
\cline { 3 - 5 } & & Yes & No & Yes \\
\hline \multirow{2}{*}{ Gender } & Male & 46 & 25 & 71 \\
\cline { 2 - 5 } & Female & 79 & 37 & 116 \\
\hline Total & & 125 & 62 & 187 \\
\hline
\end{tabular}

Table-III. Post- stratification chi square applied for gender in relation with MDR E.coli UTI cases Chi square test $=0.218 p$-value $=0.640$

When comparing frequencies of the MDR E.coli UTI cases resistant to individual groups of antibiotics, the relation was statistically significant for all the three groups individually i.e. betalactams, quinolones and aminoglycosides (with $\mathrm{p}$ value $<0.05)$. (Table-IV,V and VI)

Among the MDR E.coli UTI cases, $99 \%$ of the cases were resistant to one or more antimicrobials of the aminoglycoside family (which was statistically significant, $p=0.000$ ). Similarly, $100 \%$ cases were resistant to one or more antimicrobials of both the beta-lactams and quinolones group (which was also statistically significant, $p=0.001$ and 0.000 , respectively).

\begin{tabular}{|l|l|c|c|c|}
\hline \multicolumn{2}{|c|}{} & \multicolumn{2}{c|}{ MDR E.coli UTIs } & Total \\
\cline { 3 - 5 } & & Yes & No & Yes \\
\hline Resistant to & Yes & 124 & 4 & 128 \\
\cline { 2 - 5 } Aminoglycosides & No & 1 & 58 & 59 \\
\hline Total & & 125 & 62 & 187 \\
\hline
\end{tabular}

Table-IV. Showing relation of MDR E.coli UTI cases with cases resistant to Aminoglycosides Chi square test $=165.08 \mathrm{p}$-value $=0.000$

\begin{tabular}{|l|l|c|c|c|}
\hline \multicolumn{2}{|c|}{} & \multicolumn{2}{|c|}{ MDRE UTIs } & Total \\
\cline { 3 - 5 } \multicolumn{2}{|c|}{} & Yes & No & Yes \\
\hline Resistant to & Yes & 125 & 57 & 182 \\
\cline { 2 - 5 } Beta-lactams & No & 0 & 5 & 5 \\
\hline Total & 125 & 62 & 187 \\
\hline
\end{tabular}

Table-V. Showing relation of MDR E.coli UTI cases with cases resistant to beta-lactams Chi square test $=10.358 \mathrm{p}$-value $=0.001$

\begin{tabular}{|l|l|c|c|c|}
\hline \multicolumn{2}{|c|}{} & \multicolumn{2}{c|}{ MDRE UTIs } & Total \\
\hline \multirow{2}{*}{$\begin{array}{l}\text { Resistant to } \\
\text { Quinolones }\end{array}$} & Yes & 125 & No & Yes \\
\cline { 2 - 5 } & No & 0 & 84 & 179 \\
\hline Total & & 125 & 62 & 8 \\
\hline
\end{tabular}

Table-VI. Showing relation of MDR E.coli UTI cases with cases resistant to quinolones Chi square test $=16.850$-value $=0.000$

\section{DISCUSSION}

Urinary tract infections (UTIs) are a major burden to the health care as it is estimated that around 150 million UTIs occur yearly worldwide. ${ }^{1}$ Enterobacteriaceae, gram negative rod, being the most common agent behind all UTIs, is also becoming prevalent in Pakistan and with the development of multiple antimicrobial resistance. Choice of antimicrobial for treatment depends on the susceptibility profiles of the Enterobacteriaceae. ${ }^{4}$

The European Antimicrobial Resistance Surveillance Network (EARS-Net) have reported that antimicrobial resistance developed by $\mathrm{E}$. coli and $\mathrm{K}$. pneumonia is so much that antibiotics used as first line agents are no more effective in treating common UTIs. A survey done in 2014 in Italy reported that MDRE were mostly resistant to aminopenicillins (65\%), aminoglycosides (19\%) and fluoroquinolones (44\%). More surveys have shown that those populations with more than $25 \%$ antimicrobial resistance are of north Europe. ${ }^{14}$

In a study done by Ukah UV et al a total of 399 women were recruited; 164 women had a UTI caused by $\mathrm{E}$. coli resistant to 1 antimicrobial classes and 98 had a UTI caused by E. coli resistant to 3 antimicrobial classes. After adjustment for age, student health service (region of Canada) and either prior antibiotic use or UTI history, consumption of processed or ground chicken, cooked or raw shellfish, street foods and any organic fruit; as well as, contact with chickens, dogs and pet treats; and travel to Asia, were associated with an increased risk of UTI caused by antimicrobial resistant E. coli. A decreased risk of antimicrobial resistant UTI was associated with consumption of apples, nectarines, peppers, fresh herbs, peanuts and cooked beef. ${ }^{15}$ 
Awasthi TR, and colleagues reported in their study that among the patients presenting with urinary complaints in the outpatient department about 26\% (98/384) patients had positive urine cultures and showed bacterial growth. Similar to our study, patients presenting in his study were one third males and two third females and among them only $19 \%$ were diagnosed with culture positive UTI and among females $30 \%$ had positive urine cultures. (The difference was found statistically significant $(p<0.05)$. E. coli was the causative agent in more than half of the culture positive UTIs (53\%), second slot was occupied by K. pneumoniae (22\%), followed by Pseudomonas (12\%), Proteus (9\%) and Staphylococcus (4\%). In the treatment of these UTI patients, gentamicin and ceftriaxone was found effective in more than half of the cases nitrofurantoin in $46 \%$ cases, Cotrimoxazole (33\%), ofloxacin (30\%), nalidixic acid (16\%), and ampicillin in only $5 \%$ cases. ${ }^{16}$

In a meta-analysis, Tenney $\mathrm{J}$, et al reported that $16 / 20$ studies pointed out that the major risk factor leading to MDR microbes in urinary tract is antibiotic abuse and age; other factors includes urinary catheterization, and history of hospital stay and UTI. ${ }^{17}$ McAllister R, et al studied UTI cases reported in the residents of nursing homes. UTI was a very common presentation; mostly having pyuria $\left(14.8 \%\right.$ cases of old age). ${ }^{18}$

Castillo FR, et al reported that about 63 percent of the strains causing E. coli UTI were MDR and was more common among the females (78\%). Among these $78 \%$ cases, $40 \%$ were female children of less than 10 years and most were resistant to trimethoprim-sulfamethoxazole, ampicillin, and ampicillin-sulbactam. Most of the male cases were fluoroquinolone-resistant and MDR-phenotype. ${ }^{19}$

Unlike our study, Linsenmeyer $\mathrm{K}$, et al in his study identified more than 100 cases of MDR UTIs mostly due to Gram-negative microbes, with about $90 \%$ of males and average age was 72.8 years and $>90 \%$ cases were males. ${ }^{20}$

Ukah UV, et al reported that in during the study period of about 2 and a half years in their setting, among the positive urine cultures done, $73.8 \%$ were positive for $\mathrm{E}$. coli, with about $60 \%$ female cases, similar to our study. Among these female patients, about $24 \%$ were caused by E. coli with $24.6 \%$ MDRE UTI cases. ${ }^{21}$

Mutters NT, et al reported that among the E. coli UTI cases $54.5 \%$ (121/222) were MDRE cases $^{22}$ which was lesser than that in our study. Woodford $\mathrm{HJ}$, et $\mathrm{al}^{23}$ reported that among the diagnosed UTI patients admitted in the emergency department about $40 \%$ were not having any painful urination or frequent urination and most of these were of old age, causes behind were genital or urinary abnormalities, renal stones dehydration, and diabetes. Presenting complaints were not noted in our study as it was a retrospective study, we had limited data available.

Elsayed TI, et al reported after a detailed study that the resistance pattern of the MDRE UTI for various group of antibiotics showed that almost all were resistant to ampicillin and two third of these from Nalidixic acid; 95\% of the isolates were found to be multidrug resistant. ${ }^{24}$

Khawcharoenporn T, et al reported that in his study 62/492 diagnosed UTI patients were having nonEnterobacteriaceae UTIs and rest, 87 percent of the total patients, were having Enterobacteriaceae UTI. Median age was 44 years with a range of 14101 years with most of the cases of female gender (80\%). About $14 \%$ of these cases were having obstruction in the urinary tract like renal stone, ureteric stone, CA urinary bladder, CA vagina, or $\mathrm{BPH}$. More than half of these were having lower urinary tract infection 19\% were MDRE UTI and $81 \%$ were not multidrug resistant E. coli UTI. ${ }^{25}$

Conica E, et al concluded that fosfomycin could be taken as first-line agent against simple and recurrent UTIs, as it is safe and have targeted activity. Many studies have supported this, but more clinical trials should be done. ${ }^{26}$ Our region is endemic of MDRE UTI cases but still nitrofurantoin and fosfomycin are seen to be very effective in more than ninety percent of countries. ${ }^{27}$ 
MDR E.coli cases are increasing day by day in our country because of the fact that physicians are switching patients from intravenous antibiotics to oral antibiotics without completing the dosage and duration of one antibiotic just to facilitate the patients or some patients themselves stop the antibiotic when they are symptom free.

After all the discussion, it is clear that the choice of antibiotic in the management of MDR E.coli cases should be based on susceptibility testing and it could be the best strategy in combating these infections.

For the generation of proper guidelines for the management of MDR E.coli UTI cases its prevalence should be estimated among the population along with their susceptibility patterns; this still needs a lot of research in our country. World is moving toward rapid diagnostic tools to identify these microbes, thus guiding in the most suitable immediate treatment. ${ }^{28}$

\section{CONCLUSION}

With the rise in the microbe's resistance to multiple drugs, new therapeutic strategies are to be designed after analyzing our population's susceptibility pattern. It is not less than a challenge for physicians of a developing nation like Pakistan to treat MDR E.coli UTI cases while taking care of the cost of treatment and controlling the disease burden at the same time.

\section{CONFLICTS OF INTEREST}

The authors declare that they have no conflicts of interest.

Copyright@ 15 Sep, 2019.

\section{REFERENCES}

1. Badr AA, Shaikh GA. Recurrent urinary tract infections management in women. Sultan Qaboos Univ Med J. 2013 Aug; 13(3): 359-367.

2. Bakhsh MS, Ramazan M, Khan MG. In vitro study of various antimicrobial agents against urinary tract isolates. J Res Sci teaching. 2006; 22:28-32.

3. Elder JS, Behrman ER, Kliegman MR. "Urinary tract infection Nelson Textbook of Pediatrics". 17th ed. 2004.
4. Gupta K, Hooton TM, Naber KG. International clinical practice guidelines for the treatment of acute uncomplicated cystitis and pyelonephritis in women: A 2010 update by the infectious diseases society of America and the European society for microbiology and infectious diseases," Clini Infecti Dis, 2011: 52(5); e103-e120.

5. Hooton TM, Bradley SF, Cardenas DD, Colgan R, Geerlings SE, Rice JC, et al. Diagnosis, prevention, and treatment of catheter-associated urinary tract infection in adults: 2009 International clinical practice guidelines from the infectious diseases society of America. Clini Infect Dis 2010: 50(5):62563.

6. Sanchez GV, Master RN, Karlowsky JA, Bordon JM, "In vitro antimicrobial resistance of urinary Escherichia coli isolates among U.S. outpatients from 2000 to 2010," Antimicrobial Agents and Chemotherapy, 2012: 56(4);2181-83.

7. Qi C, Pilla V, Yu JH, Reed K. "Changing prevalence of Escherichia coli with CTX-M-type extendedspectrum $\beta$-lactamases in outpatient urinary $E$. coli between 2003 and 2008," Diagn Micro Infect Dis 2010: $67(1) ; 87-91$.

8. Gupta K, Sahm DF, Mayfield D. Antimicrobial resistance among uropathogens that cause community acquired urinary tract infections in women, a nationwide analysis. J Clini Infecti Dis. 2001; 33:89-94.

9. Ahmed MO, Clegg PD, Williams NJ, et al. Antimicrobial resistance in equine faecal Escherichia coli isolates from North West England". Inter J Clinic Microbio \& Antimicr. 2012; 9:12.

10. White GD. Antimicrobial resistance in pathogenic Escherichia coli from animals. In: Aarestrup FM, et al. editors. Antimicrobial Resistance in bacteria of animal origin. 2006. 12:145-66.

11. Paterson DL, Ko WC, Von Gottberg A. "Antibiotic therapy for Klebsiella pneumoniae bacteremia: Implications of production of extended-spectrum $\beta$-lactamases," Clini Infect Dise, 2004: 39(1); 31-7.

12. Ullah A, Shah SRH, Almugadam BS, et al. Prevalence of symptomatic urinary tract infections and antimicrobial susceptibility patterns of isolated uropathogens in Kohat Region of Pakistan. MOJ Biol Med. 2018; 3(3):85-89.

13. Abrahamian FM, Moran GJ, Talan DA. "Urinary tract infections in the emergency department," Infect Dis Clinic North America 2008: 22(1); 73-87. 
14. Mazzaroiol A, Bazaj A, Cornaglia G. Multi-drugresistant gram-negative bacteria causing urinary tract infections: A review. J Chemother 2017; 29:2-9.

15. Ukah UV, Glass M, Avery B, Daignault D, Mulvey MR, et al. Risk factors for acquisition of multidrug-resistant Escherichia coli and development of communityacquired urinary tract infections. $2017 ; 146(1): 46-57$.

16. Awasthi TR, Pant ND, Dahal PR. Prevalence of multidrug resistant bacteria in causing community acquired urinary tract infection among the patients attending outpatient department of seti zonal hospital, dhangadi, Nepal. Nepal J Biotechno. 2015; 3(1):55-9.

17. Tenney J, Hudson N, Alnifaidy H, Cheung Li JT, Fung $\mathrm{KH}$. Risk factors for acquiring multidrug-resistant organisms in urinary tract infections: A systematic literature review Saudi Pharma J. 2018; 26:678-84.

18. McAllister R, Allwood J. Recurrent multidrug resistant urinary tract infections in geriatric patients Fed Pract. 2014; 31(7):32-5.

19. Castillo FR, Moreno F, Avelar FJ, Márquez F, Harel J, Guerrero AL, et al. An evaluation of multidrug-resistant Escherichia coli isolates in urinary tract infections from Aguascalientes, Mexico: cross-sectional study. Ann Clin Microbiol Antimicrob 2018; 17:1-34.

20. Linsenmeyer K, Strymish J, Gupta K. Two simple rules for improving the accuracy of empiric treatment of multidrug-resistant urinary tract infections. Antimicrob Agents Chemother 59:7593-6.

21. Ukah UV, Glass M, Avery B, Daiganault D, Mulvey MR, Reid RJ, et al. Risk factors for acquisition of multidrugresistant escherichia coli and development of community-acquired urinary tract infections Epidemiol. Infect 2018; 146:46-57.
22. Mutters NT, Mampel A, Kropidlowski R, Biehler K, Günther $F$, Balu I, et al. Treating urinary tract infections due to MDR E. coli with Isothiocyanates - a phytotherapeutic alternative to antibiotics. Fitoterapia 2018; 129:237-40.

23. Woodford HJ, George J. Diagnosis and management of urinary tract infection in hospitalized older people. J Am Geriatr Soc. 2009 Jan; 57(1):107-14.

24. Elsayed TI, Ismail AF, Elgamal SA, Gad AH. The occurrence of multidrug resistant $E$. coli which produce ESBL and cause urinary tract infections $J$ Appl Microbiol Biochem. 2017; 1(2):8.

25. Khawcharoenporn T, Vasoo S, Singh K. Urinary tract infections due to multidrug-resistant enterobacteriaceae: Prevalence and risk factors in a Chicago emergency department Emergency Med. Intl. 2013:1-7.

26. Concia E, Bragantini D, Mazzaferri F. Clinical evaluation of guidelines and therapeutic approaches in multi drug-resistant urinary tract infections, J Chemotherapy 2017; 29:19-28.

27. Lee DS, Lee SJ, Choe HS. Community-acquired urinary tract infection by Escherichia coli in the era of antibiotic resistance. BioMed research international. $2018 ; 2018$.

28. Hawkey PM, Warren RE, Livermore DM, McNulty CA, Enoch DA, Otter JA, et al. Treatment of infections caused by multidrug-resistant Gramnegative bacteria: Report of the British Society for Antimicrobial Chemotherapy/Healthcare Infection Society/British Infection Association Joint Working Party. J Antimicrobial Chemother, 2018; 73:iii2-iii78.

\begin{tabular}{|c|c|c|c|}
\hline \multicolumn{4}{|c|}{ AUTHORSHIP AND CONTRIBUTION DECLARATION } \\
\hline Sr. \# & Author(s) Full Name & Contribution to the paper & Author(s) Signature \\
\hline 1 & Salman Azhar & $\begin{array}{l}\text { Data collection, data } \\
\text { analysis, paper writing. }\end{array}$ & \\
\hline 2 & Muhmmad Wasif Baig & $\begin{array}{l}\text { Data collection, data } \\
\text { analysis. }\end{array}$ & \\
\hline 3 & Shahid Rasool & $\begin{array}{l}\text { Data collection, data } \\
\text { analysis. }\end{array}$ & \\
\hline 4 & Rizwan Rasool Khan & Data collection. & \\
\hline 5 & Talha Munir & Paper writing & (6 \\
\hline 6 & Umber Fatima & Paper writing & \\
\hline 7 & Muhammad Ahsan & $\begin{array}{l}\text { Data entry, data analysis, } \\
\text { paper writing. }\end{array}$ & \\
\hline
\end{tabular}

